\title{
Análisis bibliométrico de las revistas de Psicología afines al ámbito Jurídico-Forense atendiendo a la WoS y el JCR (2018)
}

\section{Bibliometric analysis of Psychology journals related to the Forensic- Juridical field, taking into account the WoS and JCR (2018)}

\author{
Raúl Quevedo-Blasco $^{1}$, Alejandro Guillén-Riquelme ${ }^{2}$, Gualberto Buela-Casal $^{3}$ \\ ${ }^{1}$ Centro de Investigación Mente, Cerebro y Comportamiento (CIMCYC). Universidad de Granada. rquevedo@ugr.es \\ ${ }^{2}$ Centro de Investigación Mente, Cerebro y Comportamiento (CIMCYC). Universidad de Granada. agr@ugr.es \\ ${ }^{3}$ Centro de Investigación Mente, Cerebro y Comportamiento (CIMCYC). Universidad de Granada. gbuela@ugr.es
}

Recibido: 30/9/2019

Aceptado: 5/12/2019

Copyright (C)

Facultad de CC. de la Educación y Deporte. Universidad de Vigo

\section{Dirección de contacto:}

Raúl Quevedo-Blasco.

Facultad de Psicología. Universidad de Granada

Campus Universitario de Cartuja, s/n 18011 GRANADA (ESPAÑA)

\begin{abstract}
Resumen
Los criterios de calidad empleados por las revistas científicas, las temáticas más actuales y los autores más relevantes, son de gran interés para los investigadores de los distintos ámbitos. El objetivo principal de este estudio es proporcionar información actualizada de las revistas Jurídico-Forenses afines a la Psicología, atendiendo a los siguientes indicadores: a) Journal Citation Reports (JCR) con la última información disponible del 2018, y b) análisis bibliométrico y de contenido (del 2014 al 2018) de los artículos de las revistas objeto de estudio indexados en Web of Science (WoS). Se analizaron las 19 revistas en las categorías de Criminology \& Penology y Law en JCR, afines a la Psicología. Se incluyeron un total de 5.289 artículos. La revista con el Factor de Impacto más alto es The European Journal of Psychology Applied to Legal Context $(3,682)$. Además, es llamativo que el Factor de Impacto de las revistas analizadas ha aumentado en el 50\% de ellas, en comparación con el JCR de 2017. Se observa una amplia frecuencia del uso de términos relacionados con "crímenes" y "agresiones" en los artículos publicados en los últimos cinco años. Es importante seguir potenciando el desarrollo de revistas de calidad para la Psicología Jurídica y Forense.
\end{abstract}

\section{Palabras Clave}

Revistas Científicas, Jurídica y Forense, Journal Citation Reports, Web of Science, Análisis Bibliométrico

\section{Abstract}

The quality criteria used by scientific journals, the most current topics and the most relevant authors are of great interest to researchers in different fields. The main objective of this study is to provide updated information from forensic-legal journals related to psychology, according to the following indicators: a) Journal Citation Reports (JCR) with the latest available information from 2018, and b) bibliometric 
analysis and content (from 2014 to 2018) of the articles of the journals under study indexed in Web of Science (WoS). The 19 journals were analyzed in the categories of Criminology \& Penology and Law in JCR, related to Psychology. We included a total of 5,289 articles. The journal with the highest Impact Factor is The European Journal of Psychology Applied to Legal Context (3.682). In addition, it is remarkable that the Impact Factor of the analyzed journals has increased in the $50 \%$ of them, compared to the JCR of 2017. A wide frequency of use of terms related to "crimes" and "aggressions" is observed in articles published in the last five years. It is important to continue promoting the development of quality journals for Legal and Forensic Psychology.

\section{Key Words}

Scientific Journals, Legal and Forensic, Journal Citation Reports, Web of Science, Bibliometric Analysis

\section{INTRODUCCIÓN}

En los últimos años, conocer y analizar la producción científica (e.g., Buela-Casal, Guillén-Riquelme, Ramiro-Sánchez y Quevedo-Blasco, 2017; Carneiro-Barrera, RuizHerrera y Díaz-Román, 2019) es un aspecto de gran interés, tanto en el contexto académico, como investigador. Este interés supone un factor relevante para que incluso haya un aumento de revisiones sistemáticas que den información estructurada y actualizada sobre un tema en cuestión y en este caso, afines a la Psicología Jurídica y Forense (e.g., Cano-Montalbán y Quevedo-Blasco, 2018; Checa-Moreno y QuevedoBlasco, 2017; Selaya-Berodia, Quevedo-Blasco y Neufeld, 2018). En este sentido, y dentro del área de la Psicología Jurídica y Forense, las temáticas analizadas mediante estudios de investigación son muy variadas. A modo de ejemplo, son temas fundamentales en los últimos años el acoso, violencia y conflictos en el ámbito escolar y la victimización (e.g., Hébert, Blais y Lavoie, 2017; Jiménez y Estévez, 2017; LópezPérez, 2017; Rekalde Rodríguez, Vizcarra Morales y Macazaga, 2018), el análisis de factores asociados al noviazgo (Bringas-Molleda et al., 2017; Moral, García, Cuetos y Sirvent, 2017; Valle y Moral, 2018) y más concretamente aspectos relacionados con la violencia en el ámbito marital (Heyman et al., 2018; López-Ossorio, González Álvarez, Buquerín Pascual, García y Buela-Casal, 2017; Sjödin, Wallinius, Billstedt, Hofvander y Nilsson, 2017), siendo uno de los aspectos más importantes el abordaje de instrumentos para poder trabajar con esta problemática física, psicológica y social que se desarrolla con auge en la sociedad (Rodríguez-Díaz et al., 2017; Santirso, MartínFernández, Lila, Gracia y Terreros, 2018).

En el desarrollo de la investigación, es cada vez más relevante evaluar la calidad de las revistas científicas en las que se publica. Por ello, en países como España ha aumentado el interés por el análisis del avance de las revistas de impacto, permitiendo así conocer cómo se encuentra una determinada área de conocimiento dentro de la ciencia (Bregman, López-López y García, 2015). De hecho, no solo el número de publicaciones realizadas por un investigador, sino la calidad de las revistas en las que estos han sido publicados (ver por ejemplo Sanmarco, Vázquez y Fariña, 2019), son factores fundamentales desde hace años y, por ello, es de gran relevancia y utilidad conocer esta información.

En España, para el análisis de la calidad de las publicaciones se utilizan dos indicadores fundamentales: el Factor de Impacto del Journal Citation Reports (JCR) 
para las revistas indexadas (en Ciencias [SCIE] y Ciencias Sociales [SSCI]) en la Colección Principal de Web of Science (WoS), como el principal indicador; y, en segundo lugar, y en algunos casos de forma paralela, el Scimago Journal Rank (SJR) para las indexadas en Scopus.

Por todo lo anterior, el presente estudio tiene los siguientes objetivos: a) proporcionar información relevante y actualizada de las revistas de Criminología, Penal y Derecho (Criminology, Penology y Law) afines a la Psicología, atendiendo al último Factor de Impacto (del 2018) del Journal Citation Reports (JCR); y b) en las revistas objeto de estudio, analizar el número de artículos publicados del 2014 al 2018 en la Colección Principal de WoS, citas, países de procedencia de los autores y datos de colaboración internacional.

\section{MÉTODO}

\subsection{Materiales y unidades de análisis}

Se analizaron las revistas indexadas en el Journal Citation Reports (JCR) del 2018 (publicado el 20 de junio de 2019) en las categorías de Criminology \& Penology y Law, afines a la Psicología por estar incluidas también en alguna de las categorías de Psicología del JCR. Por otro lado, para el análisis bibliométrico y de temáticas de las publicaciones se empleó la Web of Science (WoS), limitando los años del 2014 al 2018 (ambos incluidos). La información detallada de las unidades de análisis/indicadores utilizadas en el estudio se puede ver en la Tabla 1.

\begin{tabular}{ll}
\hline Unidad de análisis/indicadores & Descripción \\
\hline Factor de Impacto (FI 2018) & $\begin{array}{l}\text { Número de citas en el año } 2018 \text { de artículos publicados } \\
\text { los dos años anteriores (2016 y 2017), dividido por el } \\
\text { número total de artículos publicados en 2016 y 2017. }\end{array}$ \\
\hline Cuartiles (Q) & $\begin{array}{l}\text { Clasificación según la posición que ocupa una revista en } \\
\text { la categoría/s en la que está indexada, atendiendo al FI: } \\
\text { Q1, Q2, Q3 y Q4. }\end{array}$ \\
\hline Frecuencia (Freq) & $\begin{array}{l}\text { Porcentaje de artículos publicados por autores de un país } \\
\text { concreto del total analizado. }\end{array}$ \\
\hline Single Country Publications (SCP) & $\begin{array}{l}\text { Número de artículos donde solo hay un autor de un país o } \\
\text { todos los autores pertenecen al mismo país. }\end{array}$ \\
\hline Multiple Country Publications (MCP) & $\begin{array}{l}\text { Número de artículos cuyos autores son al menos de dos } \\
\text { países diferentes. }\end{array}$ \\
\hline $\begin{array}{l}\text { Multiple Country Publications Ratio (MCP } \\
\text { Ratio) }\end{array}$ & $\begin{array}{l}\text { Del total de artículos de un país, el porcentaje que tienen } \\
\text { con colaboraciones internacionales. }\end{array}$ \\
\hline Frecuencia términos & $\begin{array}{l}\text { Frecuencia de aparición de términos en las "palabras } \\
\text { clave" o en los “títulos" en los artículos publicados en las } \\
\text { revistas analizadas en la Web of Science (Colección } \\
\text { Principal) en el periodo temporal de 5 años (2014-2018). }\end{array}$ \\
\hline $\begin{array}{l}\text { Análisis de las interacciones de las "palabras clave" de los } \\
\text { artículos publicados en las revistas analizadas en la Web } \\
\text { of Science (Colección Principal) en el periodo temporal de } \\
5 \text { años (2014-2018). }\end{array}$ \\
\hline
\end{tabular}

Tabla 1. Unidades de análisis/indicadores utilizadas en el estudio 


\subsection{Diseño y procedimiento}

Se trata de un estudio descriptivo mediante el análisis de documentos. La búsqueda en el JCR (para la obtención de los datos de las revistas) y en la WoS (para los datos bibliométricos y de contenido) se realizó del 20 de junio al 4 de julio de 2019. En primer lugar, se analizó la información del JCR más reciente disponible (de 2018), incluyendo tanto Science Citation Index Expanded (SCIE), como Social Sciences Citation Index (SSCI). Para depurar las revistas, el criterio fue analizar las que están indexadas en las categorías de Criminology \& Penology y/o Law, y sólo se seleccionaron las que, dentro de éstas, también estaban indexadas en una o varias categorías de Psicología del JCR (independientemente de que además estuvieran en otras categorías adicionales). Los indicadores analizados en cada revista fueron: el país de origen de cada revista, el Factor de Impacto del 2018 (y cuartil en cada una de las categorías analizadas), y la evolución del Factor de Impacto en comparación con el JCR del 2017. En segundo lugar, para el análisis de los restantes datos bibliométricos y de contenido de las revistas objeto de estudio en la WoS, se utilizó el programa R, concretamente el paquete Bibliometrix (Aria y Cuccurullo, 2017). El período temporal analizado ha sido de cinco años (de 2014 a 2018). No se incluyó el año 2019 porque en el momento de realizar los análisis no estaban (ni publicados ni indexados) en WoS todos los números de las revistas analizadas.

\section{RESULTADOS}

Se analizaron 19 revistas de Psicología en "Criminology \& Penology" y "Law atendiendo al Journal Citation Reports (2018). En la Tabla 2 se puede ver el Factor de Impacto (FI) del 2018, su comparación con el FI del 2017 y el país de edición de cada una de las revistas analizadas. Atendiendo a esto (y sin contabilizar la nueva revista incorporada en el JCR del 2018) en el 50\% de las revistas ha aumentado el FI y en el $50 \%$ ha disminuido.

Tal y como se puede ver en la Tabla 1, la revista con el Factor de Impacto (del 2018) más elevado es de España (European Journal of Psychology Applied to Legal Context). Los países que más revistas tienen indexadas son Estados Unidos y Reino Unido (con un $42,1 \%$ y un $36,8 \%$ respectivamente).

\subsection{Revistas de "Criminology \& Penology" incluidas en Psicología (JCR 2018)}

Atendiendo a datos del JCR del 2018, en la categoría Criminology \& Penology, se encuentran indexadas un total de 65 revistas, de las cuales 14 son revistas que también están en alguna de las categorías de Psicología (21,5\%). En la Tabla 3 se detallan las revistas y los cuartiles que ocupan en las categorías mencionadas anteriormente, según el Factor de Impacto del 2018. 


\begin{tabular}{|c|c|c|c|c|c|}
\hline Revistas & País & FI 2018* & Variación FI & $\begin{array}{l}\text { Criminology } \\
\text { \& Penology }\end{array}$ & Law \\
\hline $\begin{array}{l}\text { European Journal of Psychology } \\
\text { Applied to Legal Context }\end{array}$ & España & 3,682 & $\boldsymbol{4}$ & & $\mathrm{X}$ \\
\hline $\begin{array}{l}\text { Sexual Abuse-A Journal of Research } \\
\text { and Treatment }\end{array}$ & $\begin{array}{l}\text { Países } \\
\text { Bajos }\end{array}$ & 3,433 & $\downarrow$ & $\mathrm{X}$ & \\
\hline Journal of Interpersonal Violence & EE.UU. & 3,064 & 4 & $\mathrm{X}$ & \\
\hline Law and Human Behavior & EE.UU. & 2,780 & $\downarrow$ & & $\mathrm{X}$ \\
\hline Psychology of Violence & EE.UU. & 2,774 & $\mathbf{4}$ & $\mathrm{X}$ & \\
\hline Psychology Public Policy and Law & EE.UU. & 2,219 & 4 & & $\mathrm{X}$ \\
\hline Criminal Justice and Behavior & EE.UU. & 2,164 & $\downarrow$ & $\mathrm{X}$ & \\
\hline Aggression and Violent Behavior & $\begin{array}{l}\text { Reino } \\
\text { Unido }\end{array}$ & 1,983 & $\downarrow$ & $\mathrm{X}$ & \\
\hline Journal of School Violence & $\begin{array}{l}\text { Reino } \\
\text { Unido }\end{array}$ & 1,962 & $\downarrow$ & $\mathrm{X}$ & \\
\hline $\begin{array}{l}\text { International Journal of Offender } \\
\text { Therapy and Comparative } \\
\text { Criminology }\end{array}$ & EE.UU. & 1,871 & $\boldsymbol{4}$ & $\mathrm{X}$ & \\
\hline Legal and Criminological Psychology & $\begin{array}{l}\text { Reino } \\
\text { Unido }\end{array}$ & 1,764 & $\downarrow$ & $\mathrm{X}$ & $\mathrm{X}$ \\
\hline Deviant Behavior & EE.UU. & 1,490 & 4 & $\mathrm{X}$ & \\
\hline Psychology Crime \& Law & $\begin{array}{l}\text { Reino } \\
\text { Unido }\end{array}$ & 1,460 & $\downarrow$ & $\mathrm{X}$ & $\mathrm{X}$ \\
\hline $\begin{array}{l}\text { Journal of Investigative Psychology } \\
\text { and Offender Profiling }\end{array}$ & $\begin{array}{l}\text { Reino } \\
\text { Unido }\end{array}$ & 1,077 & $\boldsymbol{4}$ & $\mathrm{X}$ & \\
\hline $\begin{array}{l}\text { Journal of Forensic Psychology } \\
\text { Practice }\end{array}$ & EE.UU. & 0,872 & 4 & $\mathrm{X}$ & \\
\hline $\begin{array}{l}\text { Journal of Aggression Maltreatment } \\
\& \text { Trauma }\end{array}$ & $\begin{array}{l}\text { Reino } \\
\text { Unido }\end{array}$ & 0,833 & $\mathbf{4}$ & $\mathrm{X}$ & \\
\hline Psychiatry Psychology and Law & Australia & 0,744 & $\downarrow$ & $\mathrm{X}$ & $\mathrm{X}$ \\
\hline Behavioral Sciences \& the Law & $\begin{array}{l}\text { Reino } \\
\text { Unido }\end{array}$ & 0,682 & $\downarrow$ & & $\mathrm{X}$ \\
\hline Anuario de Psicología Jurídica & España & 0,429 & $\mathrm{Na}$ & & $\mathrm{X}$ \\
\hline
\end{tabular}

Elaboración propia. Fecha obtención de los datos: 20 de junio al 4 de julio de 2019.

Nota. EE.UU. = Estados Unidos de América; Reino Unido = Revistas solo de Inglaterra;

$\mathrm{JCR}=$ Journal Citation Reports; FI $=$ Factor de Impacto.

* Revistas ordenadas por el Factor de Impacto (FI) del 2018.

Re pistas que han aumentado el FI del 2018 (en comparación con el JCR del 2017).

Reł yistas que han bajado el FI del 2018 (en comparación con el JCR del 2017).

$\mathrm{Na}=$ No aplica: revistas incorporada en JCR del 2018 y por ello no tiene FI en 2017.

Tabla 2. Revistas de Psicología en "Criminology \& Penology" y "Law", atendiendo al JCR del 2018

\subsection{Revista de "Law" incluidas en Psicología (JCR 2018)}

En la categoría Law, atendiendo a datos del JCR del 2018 se encuentran indexadas un total de 148 revistas. El porcentaje de revistas que también están en alguna de las categorías de Psicología es menor que en la categoría Criminology \& Penology, ya que tan solo corresponde a un 5,4\%. En la Tabla 4 se detallan las revistas y los cuartiles que ocupan en las categorías analizadas en este apartado (según el Factor de Impacto del 2018). 


\begin{tabular}{|c|c|c|c|c|c|c|c|c|c|c|c|c|c|}
\hline \multirow[b]{2}{*}{ Revistas } & \multirow[b]{2}{*}{$\begin{array}{c}\text { FI } \\
\text { 2018* }\end{array}$} & \multicolumn{7}{|c|}{ PRINCIPALES CATEGORIAS ANALIZADAS } & \multicolumn{5}{|c|}{ OTRAS CATEGORÍAS } \\
\hline & & $\underset{\text { Penology }}{\text { Criminology \& }}$ & $\begin{array}{c}\text { Ps } \\
\text { Clinical }\end{array}$ & $\begin{array}{c}\text { Ps } \\
\text { Multidisciplinary }\end{array}$ & $\begin{array}{c}\text { Ps } \\
\text { Educational }\end{array}$ & $\begin{array}{c}\text { Ps } \\
\text { Developmental }\end{array}$ & $\begin{array}{c}\text { Ps } \\
\text { Applied }\end{array}$ & $\begin{array}{c}\text { Ps } \\
\text { Social }\end{array}$ & Law & $\begin{array}{c}\text { Education \& } \\
\text { Educational } \\
\text { Research }\end{array}$ & $\begin{array}{l}\text { Family } \\
\text { Studies }\end{array}$ & Sociology & Psychiatry \\
\hline $\begin{array}{l}\text { Sexual Abuse-A Journal of Research and } \\
\text { Treatment }\end{array}$ & 3,433 & $\begin{array}{c}\text { Q1 } \\
(\mathbf{5} / \mathbf{6 5})\end{array}$ & $\begin{array}{c}\text { Q1 } \\
(20 / 130)\end{array}$ & & & & & & & & & & \\
\hline Journal of Interpersonal Violence & 3,064 & $\begin{array}{c}\text { Q1 } \\
(9 / 65)\end{array}$ & & & & & $\begin{array}{c}\text { Q1 } \\
(17 / 82)\end{array}$ & & & & $\begin{array}{c}\text { Q1 } \\
(5 / 46)\end{array}$ & & \\
\hline Psychology of Violence & 2,774 & $\begin{array}{c}\text { Q1 } \\
(13 / 65)\end{array}$ & $\begin{array}{c}\mathrm{Q} 2 \\
(35 / 130) \\
\end{array}$ & & & & & & & & $\begin{array}{c}\text { Q1 } \\
(8 / 46)\end{array}$ & & \\
\hline Criminal Justice and Behavior & 2,164 & $\begin{array}{c}\text { Q2 } \\
(17 / 65)\end{array}$ & $\begin{array}{c}\mathrm{Q} 2 \\
(58 / 130)\end{array}$ & & & & & & & & & & \\
\hline Aggression and Violent Behavior & 1,983 & $\begin{array}{c}\text { Q2 } \\
(19 / 65)\end{array}$ & & $\begin{array}{c}\mathrm{Q} 2 \\
(44 / 137)\end{array}$ & & & & & & & & & \\
\hline Journal of School Violence & 1,962 & $\begin{array}{c}\text { Q2 } \\
(21 / 65)\end{array}$ & & & $\begin{array}{c}\mathrm{Q} 2 \\
(20 / 59)\end{array}$ & $\begin{array}{c}\mathrm{Q} 2 \\
(37 / 74)\end{array}$ & & & & $\begin{array}{c}\mathrm{Q} 2 \\
(66 / 243)\end{array}$ & & & \\
\hline $\begin{array}{l}\text { International Journal of Offender Therapy } \\
\text { and Comparative Criminology }\end{array}$ & 1,871 & $\begin{array}{c}\text { Q2 } \\
(22 / 65)\end{array}$ & & & & & $\begin{array}{c}\mathrm{Q} 2 \\
(41 / 82)\end{array}$ & & & & & & \\
\hline Legal and Criminological Psychology & 1,764 & $\begin{array}{c}\text { Q2 } \\
(23 / 65)\end{array}$ & & $\begin{array}{c}\text { Q2 } \\
(51 / 137)\end{array}$ & & & & & $\begin{array}{c}\text { Q1 } \\
(33 / 148)\end{array}$ & & & & \\
\hline Deviant Behavior & 1,490 & $\begin{array}{c}\text { Q2 } \\
(27 / 65)\end{array}$ & & & & & & $\begin{array}{c}\text { Q3 } \\
(37 / 63)\end{array}$ & & & & $\begin{array}{c}\mathrm{Q} 2 \\
(61 / 148)\end{array}$ & \\
\hline Psychology Crime \& Law & 1,460 & $\begin{array}{c}\mathrm{Q} 2 \\
(30 / 65)\end{array}$ & & $\begin{array}{c}\mathrm{Q} 2 \\
(65 / 137)\end{array}$ & & & & & $\begin{array}{c}\mathrm{Q} 2 \\
(52 / 148)\end{array}$ & & & & \\
\hline $\begin{array}{l}\text { Journal of Investigative Psychology and } \\
\text { Offender Profiling } \\
\end{array}$ & 1,077 & $\begin{array}{c}\mathrm{Q} 3 \\
(42 / 65)\end{array}$ & & & & & $\begin{array}{c}\text { Q4 } \\
(63 / 82) \\
\end{array}$ & & & & & & \\
\hline Journal of Forensic Psychology Practice & 0,872 & $\begin{array}{c}\mathrm{Q} 4 \\
(53 / 65)\end{array}$ & & $\begin{array}{c}\mathrm{Q} 4 \\
(103 / 137)\end{array}$ & & & & & & & & & \\
\hline $\begin{array}{l}\text { Journal of Aggression Maltreatment \& } \\
\text { Trauma }\end{array}$ & 0,833 & $\begin{array}{c}\mathrm{Q} 4 \\
(55 / 65)\end{array}$ & $\begin{array}{c}\mathrm{Q} 4 \\
(106 / 130)\end{array}$ & & & & & & & & $\begin{array}{c}\mathrm{Q} 4 \\
(35 / 46)\end{array}$ & & $\begin{array}{c}\mathrm{Q} 4 \\
(124 / 142)\end{array}$ \\
\hline Psychiatry Psychology and Law & 0,744 & $\begin{array}{c}\mathrm{Q} 4 \\
(57 / 65)\end{array}$ & & $\begin{array}{c}\mathrm{Q} 4 \\
(113 / 137)\end{array}$ & & & & & $\begin{array}{c}\mathrm{Q} 3 \\
(102 / 148)\end{array}$ & & & & $\begin{array}{c}\mathrm{Q} 4 \\
(126 / 142)\end{array}$ \\
\hline
\end{tabular}

Elaboración propia. Fecha obtención de los datos: 20 de junio al 4 de julio de 2019

Nota. $\mathrm{Q}=$ Cuartil; Ps = Psychology; FI = Factor de Impacto.

* Las revistas están ordenadas en función del Factor de Impacto (FI) del 2018.

Tabla 3. Factor de Impacto y Cuartiles de las revistas en "Criminology \& Penology” incluidas también en alguna categoría de Psicología (según el JCR del 2018) 


\begin{tabular}{|c|c|c|c|c|c|c|c|c|}
\hline \multirow[b]{2}{*}{ Revistas } & \multirow[b]{2}{*}{$\begin{array}{c}\text { FI } \\
2017^{*}\end{array}$} & \multicolumn{4}{|c|}{ PRINCIPALES CATEGORIAS ANALIZADAS } & \multicolumn{3}{|c|}{ OTRAS CATEGORÍAS } \\
\hline & & Law & $\begin{array}{c}\text { Ps } \\
\text { Multidisciplinary }\end{array}$ & $\begin{array}{c}\text { Ps } \\
\text { Applied }\end{array}$ & $\begin{array}{c}\text { Ps } \\
\text { Social }\end{array}$ & $\begin{array}{l}\text { Criminology } \\
\text { \& Penology }\end{array}$ & $\begin{array}{c}\text { Health Policy } \\
\& \text { Services }\end{array}$ & Psychiatry \\
\hline $\begin{array}{l}\text { European Journal of Psychology Applied } \\
\text { to Legal Context }\end{array}$ & 3,682 & Q1 (6/148) & $\begin{array}{c}Q 1 \\
(14 / 137) \\
\end{array}$ & & & & & \\
\hline Law and Human Behavior & 2,780 & $\begin{array}{c}\text { Q1 } \\
(11 / 148)\end{array}$ & & & $\begin{array}{c}Q 1 \\
(15 / 63)\end{array}$ & & & \\
\hline Psychology Public Policy and Law & 2,219 & $\begin{array}{c}Q 1 \\
(22 / 148)\end{array}$ & $\begin{array}{c}\text { Q2 } \\
(38 / 137)\end{array}$ & & & & $\begin{array}{c}\text { Q2 } \\
(30 / 81)\end{array}$ & \\
\hline Legal and Criminological Psychology & 1,764 & $\begin{array}{c}\text { Q1 } \\
(33 / 148)\end{array}$ & $\begin{array}{c}\mathrm{Q} 2 \\
(51 / 137)\end{array}$ & & & $\begin{array}{c}\text { Q2 } \\
(23 / 65) \\
\end{array}$ & & \\
\hline Psychology Crime \& Law & 1,460 & $\begin{array}{c}\text { Q2 } \\
(52 / 148)\end{array}$ & $\begin{array}{c}\text { Q2 } \\
(65 / 137)\end{array}$ & & & $\begin{array}{c}\mathrm{Q} 2 \\
(30 / 65)\end{array}$ & & \\
\hline Psychiatry Psychology and Law & 0,744 & $\begin{array}{c}\text { Q3 } \\
(102 / 148)\end{array}$ & $\begin{array}{c}\mathrm{Q} 4 \\
(113 / 137)\end{array}$ & & & $\begin{array}{c}\mathrm{Q} 4 \\
(57 / 65)\end{array}$ & & $\begin{array}{c}\mathrm{Q} 4 \\
(126 / 142)\end{array}$ \\
\hline Behavioral Sciences \& the Law & 0,682 & $\begin{array}{c}\text { Q3 } \\
(105 / 148) \\
\end{array}$ & & $\begin{array}{c}\mathrm{Q} 4 \\
(75 / 82)\end{array}$ & & & & \\
\hline Anuario de Psicología Jurídica & 0,429 & $\begin{array}{c}\mathrm{Q} 4 \\
(129 / 148)\end{array}$ & $\begin{array}{c}\mathrm{Q} 4 \\
(129 / 137)\end{array}$ & & & & & \\
\hline
\end{tabular}

Elaboración propia. Fecha obtención de los datos: 20 de junio al 4 de julio de 2019.

Nota. $\mathrm{Q}=$ Cuartil; Ps = Psychology; FI = Factor de Impacto.

* Las revistas están ordenadas en función del Factor de Impacto (FI) del 2018

Tabla 4. Factor de Impacto y Cuartiles de las revistas en "Law” incluidas también en alguna categoría de Psicología (según el JCR del 2018) 
3.3. Análisis bibliométricos y de contenido de las 19 revistas JCR analizadas (durante el periodo 2014-2018)

Entre el año 2014 y el 2018 en las 19 revistas analizadas se indexaron en la WoS un total de 5.289 artículos por un total de 10.664 autores (552 de los cuales publicaron 670 trabajos individuales; en los trabajos multiautoría (resto de documentos) la media de autores por documento es de 3,15). El total de documentos tiene una media de citas de 5,094 .

En la Tabla 5 se puede ver la información relativa a los países de procedencia de los autores, tipos de autorías y ratio de colaboraciones internacionales. El país con mayor número de artículos publicados en las revistas analizadas es Estados Unidos (con un $52 \%$ del total). El 83,8\% de estos artículos son de autores que pertenecen al mismo país. Los países que presentan el mayor porcentaje de colaboraciones internacionales (es decir, del total de artículos de un país el porcentaje que tienen con autores de otros países), son Suecia y China (33\% y $31 \%$ respectivamente).

\begin{tabular}{llllll}
\hline Países autoría & N $^{\mathbf{a}}$ artículos* & Freq & SCP & MCP & MCP Ratio \\
\hline Estados Unidos & 2.732 & 0,518 & 2.498 & 234 & 0,086 \\
\hline Reino Unido & 495 & 0,094 & 376 & 119 & 0,240 \\
\hline Canadá & 386 & 0,073 & 290 & 96 & 0,249 \\
\hline Australia & 360 & 0,068 & 295 & 65 & 0,181 \\
\hline Países Bajos & 202 & 0,038 & 144 & 58 & 0,287 \\
\hline España & 171 & 0,032 & 128 & 43 & 0,252 \\
\hline Israel & 105 & 0,020 & 85 & 20 & 0,191 \\
\hline Nueva Zelanda & 89 & 0,017 & 71 & 18 & 0,202 \\
\hline Suecia & 82 & 0,016 & 55 & 27 & 0,329 \\
\hline China & 74 & 0,014 & 51 & 23 & 0,311 \\
\hline
\end{tabular}

Nota. Freq $($ Frecuencia $)=$ porcentaje del total $(5,289)$ que representan los artículos de ese país; SCP (Single Country Publications) = número de artículos donde solo hay un autor o todos los autores pertenecen al mismo país; MCP (Multiple Country Publications) = número de artículos cuyos autores son al menos de dos países diferentes; MCP Ratio = del total de artículos de un país, el porcentaje que tienen con colaboraciones internacionales.

* Los datos (países) están ordenados por el número de artículos.

Tabla 5. Diez países con mayor número de artículos publicados en las revistas analizadas (datos de procedencia de los autores y otras variables asociadas).

Las palabras utilizadas más veces (2014-2018) como “palabras clave” (Keywords) fueron: intimate partner violence (285 ocurrencias), domestic violence (244), violence (204), recidivism (199), sexual assault (182), risk assessment (181), aggression (149), bullying (128), victimization (127) y trauma (121). En la Figura 1 se puede ver de forma gráfica esta información. Sin embargo, al analizar la aparición de palabras solo en los "títulos" se observa que las más frecuentes son: violence (888 ocurrencias), sexual (627), offenders (452), risk (382), partner (348), intimate (312) y mental (271). En ambos casos (palabras clave y título) se observa una amplia frecuencia del uso de términos relacionados con "crímenes" y "agresiones". 


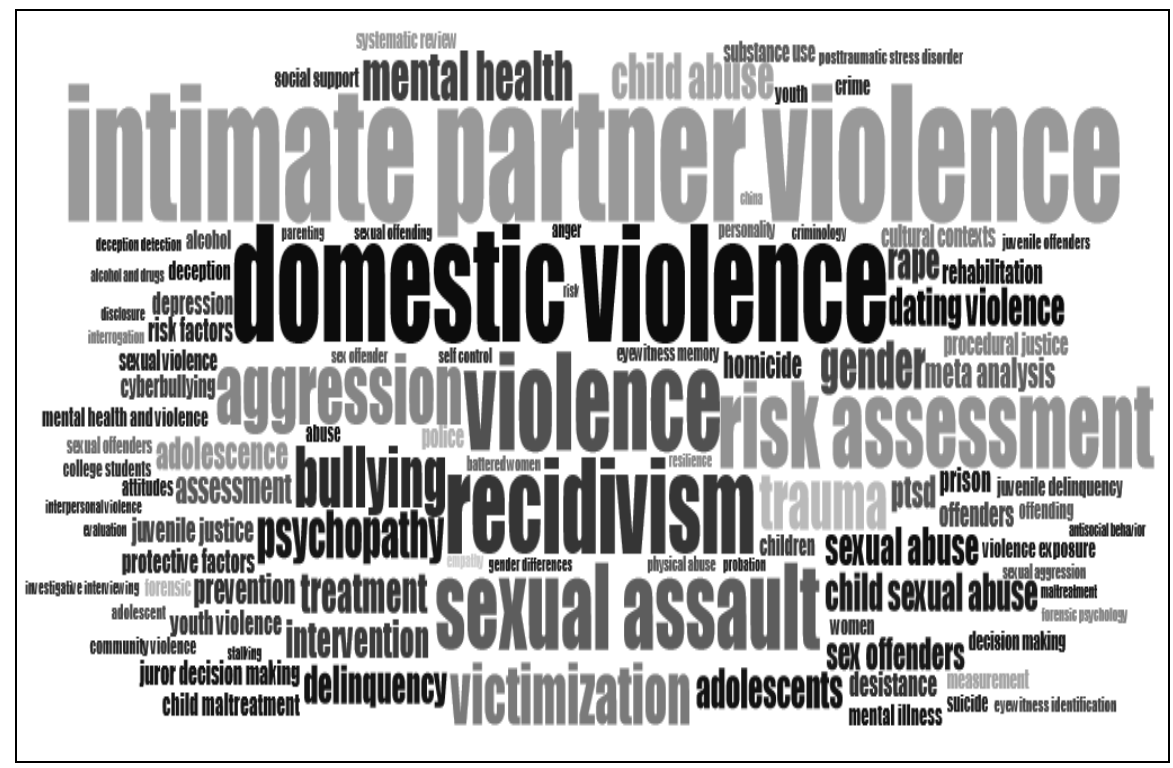

Figura 1. Frecuencia de aparición de las "palabras clave" en los artículos publicados en las revistas analizadas en la Web of Science (2014-2018)

En la Figura 2 se puede observar el análisis de redes (interacción de palabras clave), donde hay tres grandes áreas claramente definidas. La más pequeña se centraría en el estudio de la "evaluación y estudio del riesgo de reincidencias" (color verde). Las otras dos áreas más desarrolladas, estarían muy relacionas entre sí estudiando factores relacionados con "delitos violentos y agresiones" (color azul) y el "abuso sexual o agresiones domesticas" (color rojo).

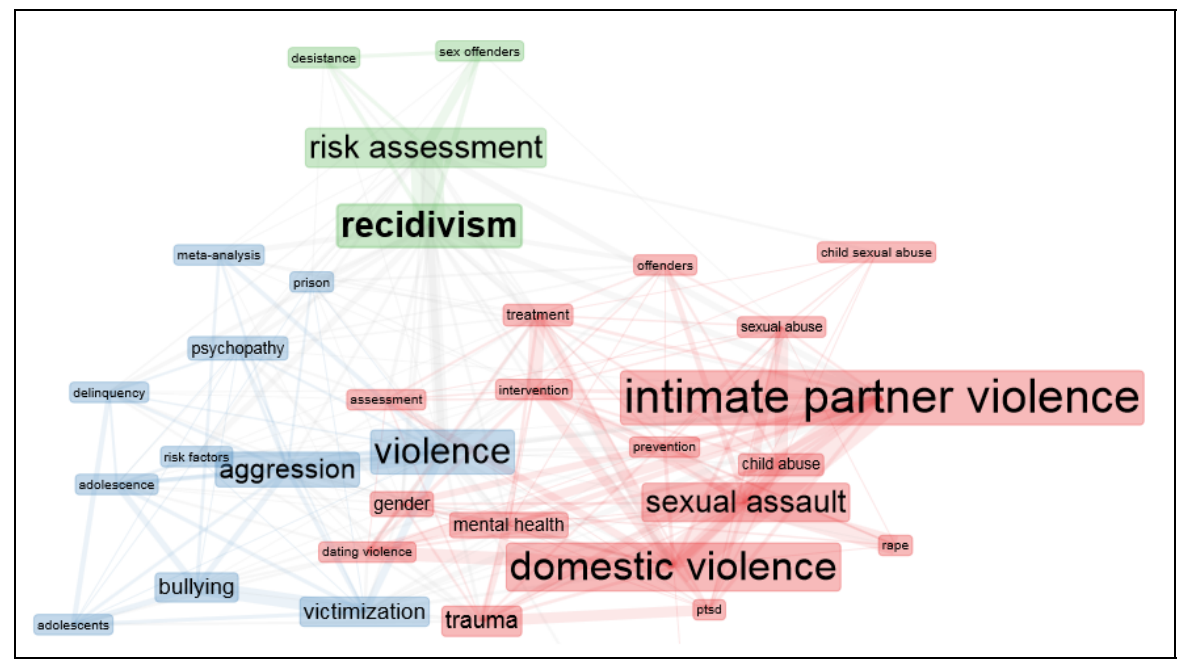

Figura 2. Análisis de redes de las "palabras clave" de los artículos publicados en las revistas analizadas en la Web of Science (2014-2018)

En la Figura 3 se puede ver otro análisis de redes, pero en este caso, de las publicaciones conjuntas entre países. Se puede apreciar que las colaboraciones entre los distintos países son significativas. 


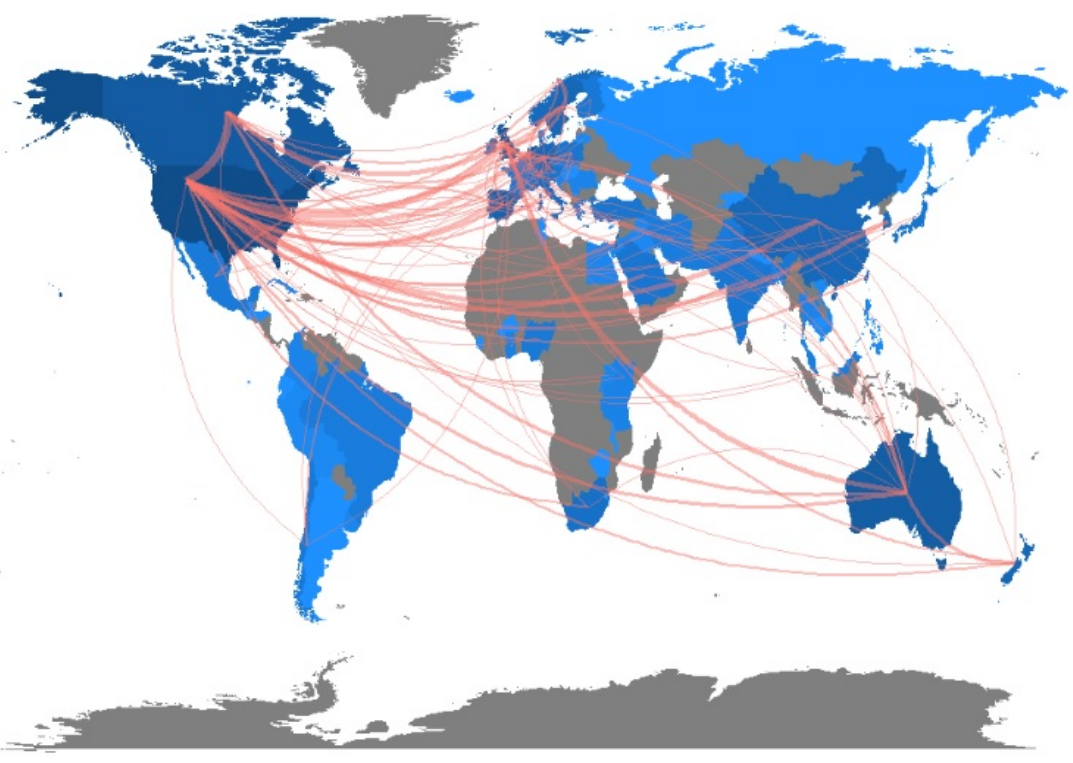

Figura 3. Mapa de redes de publicaciones conjuntas entre países (colaboraciones)

\section{DISCUSIÓN Y CONCLUSIONES}

Los estudios relevantes dentro del ámbito Jurídico y Forense como son, por ejemplo, los relacionados con aspectos afines con el abandono escolar (Hernández Prados y Alcaraz Rodríguez, 2018), la delincuencia (Llorca-Mestre, Malonda-Vidal y SamperGarcía, 2017), el maltrato infantil (Dias, Sales, Mooren, Mota-Cardoso y Kleber, 2017), el análisis de los tipos de maltratadores (Juarros-Basterretxea, Herrero, FernándezSuárez, Pérez y Rodríguez-Díaz, 2018; Manso, Guerrero-Barona y Cruz-Márquez, 2017), la violencia en las relaciones de pareja en jóvenes (Boira, Chilet-Rosell, Jaramillo-Quiroz y Reinoso, 2017), las agresiones sexuales en adolescentes (Moyano, Monge y Sierra, 2017), los programas de intervención (Carbajosa, Catalá-Minana, Lila y Gracia, 2017), etc., son fundamentales para el avance de las principales áreas de evaluación e intervención en los distintos contextos de la Psicología. El análisis de la frecuencia de los términos usados en los artículos publicados en las revistas objeto de estudio (tanto en las "palabras clave" como en los "títulos"), proporciona información muy afín con los ámbitos de aplicación de la Psicología Jurídica y Forense. Es importante resaltar que, en las revistas analizadas, a pesar de estar incluidas en categorías de "derecho y criminología", las "palabras clave" más usadas en los artículos publicados están asociadas a distintos tipos de violencia (como se puede ver en la Figura 1). Además, el análisis de redes en la Web of Science, constata la importancia de la Psicología en estas categorías, pese a que en principio se puedan percibir con menos afinidad (ver Figura 2).

Atendiendo al análisis del Journal Citation Reports (2018), los países que aportan un mayor número de revistas son Estados Unidos (8) y Reino Unido (7), suponiendo entre ambos el 78,9\% del total de revistas. Como se puede apreciar, se trata de revistas con impactos elevados, ya que el 68\% están en Q1 o Q2. Además, el Factor de Impacto ha aumentado en el 50\% de las revistas, en comparación con el JCR anterior del 2017 (ver Tabla 1).

Todo lo anterior pone de manifiesto la importancia de publicar trabajos en revistas relevantes para el área y que además tienen criterios de calidad. Por ello, analizar las 
revistas objeto de interés en este trabajo, proporcionará claridad al panorama actual atendiendo a las posibles fuentes de publicación. Existen estudios generales en los ámbitos de la Psicología y la Educación (e.g., Franco-Suárez y Quevedo-Blasco, 2017; García-Pereira y Quevedo-Blasco, 2015) pero en este trabajo, además de actualizar estudios previos (Quevedo-Blasco, 2017; Quevedo-Blasco y Martínez-Navarro, 2018) con datos del último JCR disponible (2018), se incluye un análisis bibliométrico y de contenidos (en los últimos cinco años) de las revistas analizadas.

En general, hay que tener en cuenta el matiz de la indexación de las revistas en varias categorías, ya que, en muchos casos, el cuartil es más alto en alguna otra categoría en la que está indexada (y que no siempre es Psicología). Aunque algunas de las revistas analizadas están en cuartiles altos (poniendo así de manifiesto el elevado impacto que provocan en la comunidad científica), es importante seguir potenciando el desarrollo de revistas españolas de calidad en estos ámbitos y así disponer de publicaciones de elevado impacto donde poder publicar investigaciones de calidad. Este hecho se considera de gran importancia ya que las revistas científicas son la base para el desarrollo de la investigación (Purnell y Quevedo-Blasco, 2013), sin olvidar cuestiones problemáticas derivadas del actual sistema de publicación (Buela-Casal, 2014).

Los resultados de este estudio (obtenidos tras publicarse la información del JCR del 2018) facilitan a los investigadores de estas áreas la elección de las mejores revistas para publicar (atendiendo tanto al JCR, principal criterio de calidad utilizado, como por las temáticas principales de publicación). Hay que matizar que en septiembre de cada año, Clarivate Analytics puede actualizar la información del JCR pertinente, y por ello, dependiendo del momento temporal en el que se consulten los datos, pueden variar muy ligeramente (ya que no es común que haya cambios importantes). Para futuros estudios sería importante comparar los factores de impacto y temáticas de las revistas a lo largo de los años (o al menos durante un intervalo temporal determinado), con el objetivo de analizar y entender mejor su evolución.

\section{BIBLIOGRAFÍA}

Aria, M. y Cuccurullo, C. (2017). Bibliometrix: An R-tool for comprehensive science mapping analysis. Journal of Informetrics, 11 (4), 959-975. http://dx.doi.org/10.1016/j.joi.2017.08.007

Boira, S., Chilet-Rosell, E., Jaramillo-Quiroz, S. y Reinoso, J. (2017). Sexismo, pensamientos distorsionados y violencia en las relaciones de pareja en estudiantes universitarios de Ecuador de áreas relacionadas con el bienestar y la salud. Universitas Psychologica, 16 (4), 1-12. http://dx.doi.org/10.11144/Javeriana.upsy16-4.spdv

Bregman, C., López-López, W. y García, F. (2015). La psicología clínica en las publicaciones hispanoamericanas: Un análisis bibliométrico del período 2012-2014. Terapia Psicológica, 33 (3), 205-219. Recuperado de: https://scielo.conicyt.cl/pdf/terpsicol/v33n3/art05.pdf

Bringas-Molleda, C., Estrada-Pineda, C., Suárez-Álvarez, J., Torres, A., Rodríguez-Díaz, F.J., García-Cueto, E. y Rodríguez-Franco, L. (2017). Actitud sexista y trascendente durante el noviazgo entre universitarios latinoamericanos. Revista Iberoamericana de Psicología y Salud, 8 (1), 44-55. http://dx.doi.org/10.23923/j.rips.2017.08.005

Buela-Casal, G. (2014). Pathological publishing: A new psychological disorder with legal consequences? European Journal of Psychology Applied to Legal Context, 6 (2), 91-97. http://dx.doi.org/10.1016/j.ejpal.2014.06.005

Buela-Casal, G., Guillén-Riquelme, A., Ramiro-Sánchez, T. y Quevedo-Blasco, R. (2017). Ranking de investigación de las universidades públicas españolas. Revista Iberoamericana de Psicología y Salud, 8 (1), 21-35. http://dx.doi.org/10.23923/j.rips.2017.08.003 
Cano-Montalbán, I. y Quevedo-Blasco, R. (2018). Sociodemographic Variables Most Associated with Suicidal Behaviour and Suicide Methods in Europe and America. A Systematic Review. European Journal of Psychology Applied to Legal Context, 10 (1), 15-25. http://dx.doi.org/10.5093/ejpalc2018a2

Carbajosa, P., Catalá-Minana, A., Lila, M. y Gracia, E. (2017). Differences in treatment adherence, program completion, and recidivism among batterer subtypes. European Journal of Psychology Applied to Legal Context 9 (2), 93-101. http://dx.doi.org/10.1016/j.ejpal.2017.04.001

Carneiro-Barrera, A., Ruiz-Herrera, N. y Díaz-Román, A. (2019). Tesis doctorales en Psicología tras la adaptación al Espacio Europeo de Educación Superior. Revista de Investigación en Educación, 17 (1), 32-43. Recuperado de: http://reined.webs.uvigo.es/index.php/reined/article/view/377/405

Checa-Moreno, A. y Quevedo-Blasco, R. (2017). Revisión sistemática en el Síndrome del Acento Extranjero: intervención y terapia del lenguaje. Revista Iberoamericana de Psicología y Salud, 8 (1), 1-8. http://dx.doi.org/10.23923/j.rips.2017.08.001

Dias, A., Sales, L., Mooren, T., Mota-Cardoso, R. y Kleber, R. (2017). Child maltreatment, revictimization and Post Traumatic Stress Disorder among adults in a community sample. International Journal of Clinical and Health Psychology, 17 (2), 97-106. http://dx.doi.org/10.1016/j.ijchp.2017.03.003

Franco-Suárez, O. y Quevedo-Blasco, R. (2017). Análisis de las revistas iberoamericanas de Psicología y de Educación indexadas en el Journal Citation Reports del 2015. PSIENCIA. Revista Latinoamericana de Ciencia Psicológica, 9 (4), 1-23. http://dx.doi.org/10.5872/psiencia/9.4.22

García-Pereira, S. y Quevedo-Blasco, R. (2015). Análisis de las revistas iberoamericanas de Psicología y de Educación indexadas en el Journal Citation Reports de 2013. European Journal of Education and Psychology, 8 (2), 85-96. Recuperado de: http://www.elsevier.es/es-revista-european-journal-education-psychology-235-pdfS1888899215000069

Hébert, M., Blais, M. y Lavoie, F. (2017). Prevalence of teen dating victimization among a representative sample of high school students in Quebec. International Journal of Clinical and Health Psychology, 17 (3), 225-233. http://dx.doi.org/0.1016/j.ijchp.2017.06.001

Hernández Prados, M. y Alcaraz Rodríguez, M. (2018). Factores incidentes en el abandono escolar prematuro. Revista de Investigación en Educación, 16 (2), 182-195. Recuperado de: http://reined.webs.uvigo.es/index.php/reined/article/view/346

Heyman, R.E., Kogan, C.S., Foran, H.M., Burns, S.C., Smith Slep, A.M., Wojda, A.K., Keeley, J.W., Rebello, T.J. y Reed, G.M. (2018). A case-controlled field study evaluating ICD-11 proposals for relational problems and intimate partner violence. International Journal of Clinical and Health Psychology, 18 (2), 113-123. http://dx.doi.org/10.1016/j.ijchp.2018.03.001

Jiménez, T.I. y Estévez, E. (2017). School aggression in adolescence: Examining the role of individual, family and school variables. International Journal of Clinical and Health Psychology, 17 (3), 251-260. http://dx.doi.org/10.1016/j.ijchp.2017.07.002

Juarros-Basterretxea, J., Herrero, J., Fernández-Suárez, A., Pérez, B. y Rodríguez-Díaz, F.J. (2018). Are generalist batterers different from generally extra-family violent men? A study among imprisoned male violent offenders. European Journal of Psychology Applied to Legal Context, 10 (1), 8-14. http://dx.doi.org/10.5093/ejpalc2018a1

Llorca-Mestre, A., Malonda-Vidal, E. y Samper-García, P. (2017). Prosocial reasoning and emotions in young offenders and non-offenders. European Journal of Psychology Applied to Legal Context, 9 (2), 65-73. http://dx.doi.org/10.1016/j.ejpal.2017.01.001

López-Ossorio, J.J., González Álvarez, J.L., Buquerín Pascual, S. García, L.F. y Buela-Casal, G. (2017). Risk factors related to intimate partner violence police recidivism in Spain. International Journal of Clinical and Health Psychology, 17 (2), 107119. http://dx.doi.org/10.1016/j.ijchp.2016.12.001 
López-Pérez, M.G. (2017). Acoso escolar y cibernético en estudiantes universitarios. Revista de Investigación en Educación, 15 (1), 11-26. Recuperado de: http://reined.webs.uvigo.es/index.php/reined/article/view/200/197

Manso, J.M., Guerrero-Barona, E.G. y Cruz-Márquez, B. (2017). Actitudes sexistas y asunción de responsabilidad en agresores condenados a prisión por violencia de género en España durante los años 2012 y 2013. Universitas Psychologica, 16 (3), 1-13. http://dx.doi.org/10.11144/Javeriana.upsy16-3.asar

Moral, M.V., García, A., Cuetos, G. y Sirvent, C. (2017). Violencia en el noviazgo, dependencia emocional y autoestima en adolescentes y jóvenes españoles. Revista Iberoamericana de Psicología y Salud, 8 (2), 96-107. http://dx.doi.org/10.23923/j.rips.2017.08.009

Moyano, N., Monge, F.S. y Sierra, J.C. (2017). Predictors of sexual aggression in adolescents: Gender dominance vs. rape supportive attitudes. European Journal of Psychology Applied to Legal Context, 9 (1), 25-31. http://dx.doi.org/10.1016/j.ejpal.2016.06.001

Purnell, P.J. y Quevedo-Blasco, R. (2013). Benefits to the Spanish research community of regional content expansion in Web of Science. International Journal of Clinical and Health Psychology, 13 (2), 147-154. Recuperado de: http://www.elsevier.es/en-revistainternational-journal-clinical-health-psychology-355-pdf-X1697260013005067

Quevedo-Blasco, R. (2017). Revistas de Psicología en Criminología, Penología y Derecho atendiendo al JCR. En E. Arias, T. Corrás, B.G. Amado y R. Gallego (Eds.). X Congreso (Inter) Nacional de Psicología Jurídica y Forense. Libro de Actas (pp. 439-442). Sevilla: Dirección General de Violencia de Género, Consejería de Igualdad y Políticas Sociales, Junta de Andalucía.

Quevedo-Blasco, R. y Martínez-Navarro, E. (2018). Revistas Jurídico-Forenses afines a la Psicología atendiendo al JCR de 2017. En E. Arias, J. Sanmarco y X. Camplá (Eds.). XI Congreso (Inter) Nacional de Psicología Jurídica y Forense. Libro de Actas (pp. 491-493). Santiago de Compostela: Sociedad Española de Psicología Jurídica y Forense.

Rekalde Rodríguez, I., Vizcarra Morales, M.T. y Macazaga, A.M. (2018). Indagando en nuevas líneas de acción ante los conflictos escolares: Las comunidades de aprendizaje en constante revisión. Revista de Investigación en Educación, 16 (2), 170-181. Recuperado de: http://reined.webs.uvigo.es/index.php/reined/article/view/345

Rodríguez-Díaz, F.J., Herrero, J., Rodríguez-Franco, L., Bringas-Molleda, C., Paíno-Quesada, S.G. y Pérez, B. (2017). Validation of Dating Violence Questionnarie-R (DVQ-R). International Journal of Clinical and Health Psychology, 17 (1), 77-84. http://dx.doi.org/10.1016/j.ijchp.2016.09.001

Sanmarco, J., Vázquez, M.J. y Fariña, F. (2019). Comparación de los índices de citas y clasificación de revistas del Journal Citation Reports y Scopus en el campo de la Psicología. Revista Iberoamericana de Psicología y Salud, 10 (2), 122-134. http://dx.doi.org/10.23923/j.rips.2019.02.030

Santirso, F.A., Martín-Fernández, M., Lila, M., Gracia, E. y Terreros, E. (2018). Validation of the Working Alliance Inventory-Observer Short Version with male intimate partner violence offenders. International Journal of Clinical and Health Psychology, 18 (2), 152-161. http://dx.doi.org/10.1016/j.ijchp.2018.02.003

Selaya-Berodia, A., Quevedo-Blasco, R. y Neufeld, C.B. (2018). Personalidad, estado emocional y ansiedad: Una revisión sistemática de su influencia sobre las falsas memorias. Revista Mexicana de Psicología, 35 (1), 5-25. Recuperado de: http://comeppsi.com/images/rmp/RMP\%20351\%20005-025.pdf

Sjödin, A-K., Wallinius, M., Billstedt, E., Hofvander, B. y Nilsson, T. (2017). Dating violence compared to other types of violence: similar offenders but different victims. European Journal of Psychology Applied to Legal Context, 9 (2), 83-91. http://dx.doi.org/10.1016/j.ejpal.2017.03.001 
Quevedo-Blasco, R., Guillén Riquelme, A. y Buela-Casal, G.

Valle, L. y Moral, M.V. (2018). Dependencia emocional y estilo de apego adulto en las relaciones de noviazgo en jóvenes españoles. Revista Iberoamericana de Psicología y Salud, 9 (1), 27 -

41. http://dx.doi.org/10.23923/j.rips.2018.01.013 